\title{
Evolución de la evaluación externa de los estudiantes en la educación básica colombiana*
}

\author{
Evolution of the external evaluation of basic \\ education students in Colombia
}

\author{
Tulio Ramírez** \\ Gloria Amparo Orrego Agudelo**
}

RECIBIDO: 2 DE JULIO DE 2012

APROBADO: 15 DE OCTUBRE DE 2012

Resumen. Se presenta de forma descriptiva la evolución de la evaluación en los niveles básica primaria y secundaria de los estudiantes colombianos, teniendo como propósito presentar lo que ha sido el trascurrir de la misma y la importancia que el gobierno nacional le ha

Artículo de revisión resultado del curso de Seminario de Mitos y Realidades Educativas del Doctorado en Educación en Universidad Central de Venezuela (UCV).

** Sociólogo y abogado, Universidad Central de Venezuela (Venezuela), Magister en Formación de Recursos Humanos (Universidad Católica Andrés Bello, Venezuela), Doctor en Filosofía y Ciencias de la Educación UNED-España (2006). Actualmente se desempeña como Coordinador del Doctorado en Educación de la Universidad Central de Venezuela. Gerente de Desarrollo Docente y Estudiantil del Vicerrectorado Académico de la Universidad Central de Venezuela (Caracas-Venezuela). Coordinador de la línea de investigación sobre textos escolares del Doctorado en Educación de la Universidad Central de Venezuela. E-mail: tuliorc1@gmail.com

*** Economista, Universidad Cooperativa de Colombia (UCC), Seccional Bucaramanga. Especialista en Administración y Docencia Universitaria, Universidad de Santander (UDES). Especialista en Teoría, Métodos y Técnicas de Investigación Social. Universidad Industrial de Santander-UIS. Doctoranda en Educación, Universidad Central de Venezuela (UCV). Actualmente es docente-investigadora de la Facultad de Comunicaciones, Artes y Diseño. Programa de Mercadeo y Publicidad y DocenteCoordinadora de Prácticas Empresariales. Facultad de Comunicaciones, Artes y Diseño. Programa de Mercadeo y Publicidad. Universidad de Santander (UDES), Colombia. Líder del Grupo de Investigación Centro de Investigaciones de Mercadeo y Publicidad. E-mail: gaorrego@udes.edu.co y gloriaaorrego@yahoo.com

ITINERARIO EDUCATIVO • ISSN OILI-2753 • AÑO XXVI, N. ${ }^{\circ} 60$ • ENERO - JUNIO DE 2OIL • P. 27-54 
venido otorgando. Por tanto se parte del origen y conceptualización de la evaluación, la evolución y organización del sistema evaluativo colombiano, al igual que los aprendizajes al interior de las aulas; Se muestra el proceso de evaluación y las medidas que cada uno de los períodos de gobierno adoptaron en el tema, dada la importancia y las exigencias de los organismos internacionales que orientaron en este sentido, con miras en la promoción de una cultura evaluativa y la calidad de la educación. Finalmente se puede inferir que el sistema educativo colombiano ha venido construyendo a través del tiempo un sistema evaluativo que inicialmente propende por la obtención de información válida y confiable que le permita el inicio de ajustes al mismo, como también al mejoramiento del sistema educativo, como lo expresa en la normatividad emitida, pero que sin lugar a dudas este proceso genera debate entre las comunidades educativas y aún tiene mucho camino por recorrer.

Palabras clave. Sistema de Evaluación, Educación Básica, Competencias, Estudiantes, Calidad de la Educación.

Abstract. In this text, it is presented in detail, the evolution of the assessment system for students in Colombian basic education: primary and secondary. The purpose is to present what it has been the elapse of the mentioned evolution, and the importance that the national government has been giving to it. As a result, the text starts with the origin and conceptualization of the assessment system, the development and organization of Colombian assessment system, as well as, the learning processes within the classroom. It is followed by mentioning the process of assessment and grading systems that each government period adopted on this issue, due to the value and requirements of international organizations that orientated in this regards, in order to encourage an awareness of the assessment system and quality of education. Finally, it is inferred that, as mentioned in issued regulations, Colombian system education has been building over time an assessment system, that initially, aims for obtaining valid and reliable information that allows to perform adjustments to it, as well as, to improve the educational system. However, there is no doubt this process generates debate among educational communities and still has long way to go.

Keywords. Assessment System, basic education, skills, students, quality education. 


\section{Introducción}

En diversos países latinoamericanos se han implementado un conjunto de orientaciones en materia de calidad de la educación. El gobierno colombiano, con el fin de adaptar los métodos de evaluación que permitan dar cuenta de la calidad educativa en el país, ha estado al tanto de estas orientaciones y su impacto real en el logro de una mejor educación. Esta preocupación ha traído como consecuencia que las políticas educativas nacionales de los últimos años enfaticen en la importancia de la evaluación de los estudiantes, como mecanismo que permita conocer qué tanto ha aprendido el alumnado en diferentes momentos de los ciclos escolares y cuáles son los factores que inciden en estos resultados. Las autoridades educativas colombianas han entendido que de esta manera se puede establecer la calidad de la educación que están recibiendo los estudiantes, además de permitir tomar las decisiones que permitan el perfeccionamiento de los procesos curriculares, pedagógicos y de gestión (Plan Decenal de Educación, 2006-2016).

En ese sentido, el presente artículo, pretende mostrar la evolución de la evaluación de la educación básica primaria y secundaria colombiana. En primera instancia se esboza el origen y conceptualización de la evaluación tanto en el ámbito nacional como en el internacional, presentando la evolución y organización del proceso evaluativo de dichos niveles educativos en Colombia y el fundamento de las diferentes pruebas que se vienen aplicando en los niveles de primaria, secundaria y educación superior; posteriormente, se expone la normatividad de la evaluación al interior de las aulas.

En la realización de este documento se han tenido en cuenta fuentes primarias como normas, documentos oficiales, así como portales virtuales de organismos oficiales que ofrecen información y datos sobre el tema objeto de estudio. De igual manera se utilizaron como fuentes secundarias, autores tanto colombianos como extranjeros, cuyos aportes en el área de la evaluación permiten una comprensión más cabal de la problemática.

Colombia muestra importantes avances en la construcción de un sistema evaluativo que le ha permitido hacer reformas de gran envergadura en la dinámica de los aprendizajes de los estudiantes. Con esto se continúa consolidando este sistema, buscando permanentemente la construcción 
de la cultura evaluativa que redunde en el mejoramiento en la calidad de la educación.

\section{La evaluación de la Educación Básica en Colombia}

\section{Origen y conceptualización de la evaluación}

Los requerimientos que conlleva la globalización, nos obligan a reconocer que el conocimiento se convierte en el factor de producción de la vida social, la ciencia, la cultura y por supuesto la educación, pues sus dimensiones traspasaron lo que por tradición han sido históricamente, debido a que el conocimiento es hoy, no solo el instrumento para explicar y comprender la realidad, si no también en motor de desarrollo y cambio social. (Drucker, P.F, 1996, p.28).

La globalización y la sociedad del conocimiento han invadido a la mayoría de los países, afectando a la economía, la cultura y la educación. Es así como los conceptos de calidad, excelencia, logros y eficiencia se convierten en los parámetros que miden el éxito de la economía y de las acciones políticas tanto en los países que han logrado mejorar sus estándares de vida como en aquéllos que se encuentran en vías de desarrollo. La impronta de la globalización y la sociedad del conocimiento han impulsado la modificación de las organizaciones estatales de la educación, la concepción del trabajo y la economía, estableciéndose una sinergia entre estos factores que ha potenciado el desarrollo de la sociedad. Así entonces, en palabras de Cerda \& León (2005, p.11), aspectos como logros, competencias, gestión de calidad, excelencia, estándares de calidad, insumos, entre otros, que actualmente son familiares en el campo educativo, han sido categorías propias del sector empresarial dejando ver que entre estos dos sectores existe una estrecha relación.

Los autores referidos anteriormente afirman que Colombia mantiene relaciones económicas, tecnológicas y culturales con diversidad de países y recibe directrices de organismos como el Banco Mundial, el Banco Interamericano de Desarrollo, el Fondo Monetario Internacional, la Organización de Cooperación y Desarrollo Económico (OCDE) y la Organización de las Naciones Unidas para la Educación (UNESCO) entre otros, situación que hace que dichas orientaciones sean adaptadas para dar respuesta a las demandas de eficiencia, eficacia y efectividad, 
tal y como lo indican los organismos internacionales antes mencionados (Cerda y León, 2005, p.12).

Según Brunner (2002), la calidad es un concepto socialmente construido e históricamente determinado, por tanto responde a las exigencias del sistema económico, social, político y cultural de cada nación. Independientemente del concepto que se tenga de la calidad de la educación es necesario tener información unificada sobre el cumplimiento de los objetivos acordados por los organismos financiadores del sistema educativo en su conjunto. Las exigencias internacionales comprometieron al país a crear su propio sistema de evaluación de la calidad en las instituciones de educación superior en sus inicios, con miras a extenderse en todo el sistema educativo colombiano. Este sistema fue creado por la Ley 30 de 1992, de tal manera que le permitiera medir y hacer seguimiento a través de escalas censales y muestrales, y con ello, determinar los niveles de desarrollo de las competencias básicas logradas y de las diferentes áreas del conocimiento, lo que permitió identificar condiciones de valoración de la calidad en los niveles de primaria y secundaria, aspecto este que se tratará más adelante.

En estos términos, para hablar de calidad ${ }^{1}$ implica necesariamente introducir el concepto de evaluación. Restrepo (1974) plantea que la evaluación consiste en la apreciación de un objeto, persona, atributo, programa, institución o sistema, a partir de ciertos criterios y destaca el aspecto valorativo como elemento que objetiviza la escogencia de factores o indicadores relacionados con el propósito de lo que se evalúa. Ahora bien, como toda actividad humana, es social y la mayoría institucional, y cualquier sistema social está influenciado y determinado por variables circundantes, es en consideración de todas estas circunstancias como aparecen las conceptualizaciones teóricas y los modelos de evaluación.

Según Correa, Puerta \& Restrepo (2002, p.28), por diferentes que sean las maneras de concebir el fin y los métodos de la evaluación que estas

1 Se trae aquí una de las definiciones más comunes de la calidad en general desde el ámbito empresarial con el propósito de aclarar que el término nace en este campo del conocimiento y ha debido se ha venido adaptando al campo educativo por tanto la «Calidad es la satisfacción de las necesidades y expectativas de los clientes». En los últimos años esta definición ha «evolucionado» incluyendo la noción de racionalidad: «Calidad es satisfacer las necesidades de los clientes y sus expectativas razonables» (López, 1997, p.43). 
definiciones implican, todas podemos reducirlas a un común denominador: el de que todas se refieren a la investigación y la apreciación de la eficiencia, eficacia, efectividad y relevancia social de una institución, programa o proyecto, para lo cual se requiere disponer de información sobre los insumos, los procesos y los productos o resultados (Stufflebean \& Shinkfield, 1995, p. 94). Cuanto más válidas, confiables y representativas sean estas informaciones, mayor será la posibilidad de reorganizar los fines y los medios de un programa o proyecto, de tal manera que éste arroje resultados óptimos. Obtener y valorar estas informaciones es lo que denominamos evaluación.

En el mundo, la evaluación programas tuvo sus orígenes en Estados Unidos e Inglaterra y hoy sus avances son de gran notoriedad en Europa, Australia, Israel, África y América Latina (Worthen, Sanders \& Fitzpatrick, 1997).

En educación, que por su razón de ser siempre ha incluido la actividad evaluativa o de comprobación como componente esencial, la evaluación de programas comenzó a figurar como una «positividad», o sea, un campo con cierto objeto más allá de la evaluación del aprendizaje académico, a partir de los trabajos de Ralph Tyler quien, desde 1934, planteó la necesidad de transformar el sistema de evaluación incluyendo aspectos filosóficos, culturales y escolares para constatar logros de objetivos. Tyler acuñó el término «evaluación» para expresar una apreciación del rendimiento estudiantil más holística, más allá de la era del «examen» y de lo académico, para incluir lo contextual de los programas educativos y de su rendimiento.

Hasta finales de la década de 1950, la orientación tyleriana de una evaluación basada en comprobación del logro de objetivos no solo se usó en la educación sino también en los programas sociales. Hechos políticos y teóricos como la influencia de la psicología congnoscitivista, en particular los trabajos de Piaget, Bruner y Ausubel, al insistir en el desarrollo y evaluación de las funciones intelectuales primarias, el aprendizaje por descubrimiento y el aprendizaje significativo, demandaron una evaluación más orientada a procesos que a resultados comparados con objetivos. Este cambio en la teoría del aprendizaje puntualiza, en el campo de la evaluación institucional y de programas, un viraje de la valoración de productos hacia la comprensión y mejoramiento de procesos, concepción que se evidenciará en el florecimiento de modelos cualitativos a partir 
de la década de 1960. Así, los métodos cualitativos entran a compartir con los cuantitativos la implementación de la evaluación.

Desde el punto de vista político, el sentimiento de culpa interiorizado en Estados Unidos sobre la baja calidad de su educación, por el efecto demostración del triunfo espacial soviético que lanzó el primer Sputnik en 1959, intensificó la conciencia de la necesidad de evaluar todo proyecto educativo antes de renovarle fondos federales a los Distritos Educativos, y de exigir evidencias evaluativas en planes y propuestas innovadoras. Tal necesidad se materializó en una norma legal, el Título I del Acta de Educación Secundaria de 1964, que incluía requisitos específicos de evaluación de programas como criterio para renovar fondos ofrecidos a los distritos educativos para acelerar el mejoramiento de la educación (Madauss y Linnan, 1983, p. 53). La inexistencia de procedimientos apropiados para el seguimiento y evaluación de proyectos sociales despertó el interés por la elaboración y refinamiento de modelos y procedimientos evaluativos diferentes a los subyacentes en el examen tradicional, esto es, distintos al simple cuestionario basado en objetivos.

Se ensayó, entonces, el método experimental a través de diseños experimentales y cuasi experimentales, aplicados a programas de educación, salud y bienestar social (Suchman, 1967 y Campbell, 1969). Con estos diseños el Banco Mundial ha evaluado, desde hace varias décadas hasta hoy día, proyectos en áreas sociales (Grossman, 1994 y Newman, Rawlings \& Gertler, 1994). El interés de este tipo de evaluación estuvo centrado, desde los años 70 al 90, en evaluar las repercusiones sociales o el impacto real de la inversión en los programas sociales financiados por este organismo. Pero pronto se hizo obvio que este enfoque, si bien lograba evidencias certeras sobre efectos, debido a determinados tratamientos o intervenciones transformadoras, dejaba por fuera elementos no verificables experimentalmente, pero de necesaria apreciación cuando de mejorar procesos se trata.

En la década de los setenta se promueven grupos de expertos dedicados a la evaluación. Así, se crean asociaciones profesionales en Estados Unidos, Canadá, Australia y Europa, como la American Evaluation Association, la Canadian Evaluation Society, la Sociedad Europea de Evaluación (creada en 1994), la Australasian Evaluation Society (Worthen, Sanders, and Fitzpatrick, 1997), la African Evaluation Association, la United Kingdom Evaluation Society, y la Assocciazione Italiana de 
Valutazione. De igual manera se crean se crean publicaciones periódicas especializadas, como The American Journal of Evaluation (antes Evaluation Practice) y New Directions For Evaluation, de la Asociación Americana de Evaluación, de carácter general, Educational Evaluation and Policy Analysis, de la Asociación Americana de Investigación en Educación, más específica, Canadian Journal of Program Evaluation, y Evaluation (de la Asociación Europea), se establecen redes electrónicas de evaluadores e investigadores, como XC-Eval y Evaltalkt; se llevan a cabo Cyberconferencias como la Primera Cyberconferencia Internacional de Evaluación, celebrada en 1998 con la participación de 25 países, entre ellos Colombia; se organizan posgrados en evaluación de programas y se refinan modelos evaluativos, apareciendo, además, nuevas propuestas con gran despliegue y acogida mundial, como es el caso de los modelos de evaluación de contexto, insumos, proceso y producto, que empezó siendo un modelo de evaluación de programas en educación, pero que en sucesivas actualizaciones se proyectó como un modelo para evaluar la educación continuada en Medicina, para evaluar programas de desarrollo comunitario y de servicio social (Stufflebeam, 1997, citado por Correa, Puerta \& Restrepo, 2002, p. 57).

A partir de este empuje intelectual y académico se hizo el esfuerzo por fundamentar epistemológicamente los enfoques evaluativos existentes, tanto los experimentales clásicos y sus afines, más orientados a evaluar productos, como los más cualitativos, empeñados en apreciaciones más relacionadas con los procesos de implementación de los programas y las necesidades de los interesados. Los objetivos de la evaluación en la primera etapa de la historia de esta disciplina fueron los alcanzados en el programa de instrucción, inicialmente, extendidos luego a otras áreas del sector social, es decir, comparar resultados frente a objetivos propuestos. En la segunda etapa, junto con los productos, se resaltó la importancia de los procesos y más recientemente, durante la tercera etapa, se incluyeron el contexto, los esfuerzos, insumos o estrategias generales de implementación, y los procesos y resultados organizacionales, internos y de impacto en la sociedad. Se pasó así, poco a poco, de una evaluación meramente académica o de aprendizajes, a la evaluación de programas y de instituciones (Correa, Puerta \& Restrepo, 2002, p. 59).

Lo planteado permite ver cómo desde el ámbito académico ha ido evolucionando del concepto de evaluación de la educación como disciplina, con miras a determinar condiciones que permitan evaluar los resultados 
del proceso educativo con miras a contribuir con el mejoramiento de su calidad.

\section{Evolución y organización del proceso evaluativo de la Educación Básica Primaria y Secundaria colombiana}

La educación formal colombiana se ofrece de manera pública (las entidades oficiales o del Estado) y privada (instituciones de los particulares). Está conformada por cuatro niveles: a) el preescolar o grado cero (párvulos, pre-jardín, jardín y transición); b) la Educación Básica, comprendida por dos ciclos: la básica primaria con los grados primero a quinto, y la básica secundaria, con los grados de sexto a noveno; c) la educación media comprende los grados décimo a once y d) la Educación Superior, conformada por los estudios de pregrado (cinco años promedio), las especializaciones o posgrados (uno o año y medio), las maestrías de dos años y los doctorados de tres y cuatro años. Este último nivel es ofrecido no solo por las instituciones de educación superior del sector público, también es ofrecido por corporaciones, fundaciones y universidades privadas, como consecuencia de la política de recortes presupuestales adelantada desde hace varios años por el Estado, lo que ha permitido la proliferación de la oferta privada.

Hecha la descripción anterior, aboquemos ahora el tema central de este artículo: la evaluación. Empecemos diciendo que en Colombia, esta ha sido objeto de preocupación dadas las reformas educativas promovidas a partir del período 1986-1990, durante el gobierno del Presidente Virgilio Barco Vargas. Esto debido a la coyuntura del proceso de descentralización administrativa a comienzos de la década, de la nueva Constitución de 1991, y luego la expedición de la Ley 115 de 1994 o Ley General de la Educación, pues solo hasta entonces, el Gobierno le da tratamiento a la calidad de la educación y la tiene en cuenta dentro de las reformas de sus políticas.

En Colombia las transformaciones en el tema de políticas oficiales educativas habían sido consecuencia de las reformas introducidas y marcadas en cierta forma por los intereses de los partidos políticos liberal y conservador. Sin embargo, más allá de de las intenciones partidarias, temas como la cobertura, el presupuesto la expansión educativa entre otras reformas, debieron ser introducidas gracias al período de industrialización vivido por el país a mediados del siglo XX, al pasar de un país rural 
a un país urbano. Importante anotar que las demandas internacionales de la época obligaron al país a reflexionar en este sentido, además del atraso económico en que se encontraba la nación para ese momento.

Los organismos internacionales comenzaron a exigir a los gobiernos e instituciones públicas sin ánimo de lucro, la inclusión de componentes evaluativos para el seguimiento de los programas y la rendición de cuentas no solo a las entidades financiadoras, sino también a la comunidad. La exigencia pasaba por la elaboración de modelos sistemáticos que detectaran la efectividad de los programas, modelos que iban desde los diseños experimentales y cuasi experimentales hasta la adopción o adaptación de modelos empírico analíticos y cualitativos construidos y sustentados por los expertos en evaluación.

El Instituto Colombiano de Pedagogía (ICOLPE), llevó a cabo seminarios y eventos de preparación de personal para evaluar diversos las instituciones y los programas de estudio. Es así como entre 1971 y 1972, elaboró los criterios y escalas de evaluación de textos (ICOLPE, 1972) y, entre 1973 y 1975, adelantó seminarios sobre evaluación institucional y de programas. El Instituto Colombiano para el Fomento de la Educación Superior (ICFES), por su parte, entró en la década de 1970 a manejar modelos de evaluación reconocidos, como los de Stufflebeam y otros, para fundamentar su actividad evaluadora.

En 1986, la UNESCO, a través de un convenio de cooperación con el Banco Mundial, realizó un importante estudio evaluativo de los aspectos de la calidad de la enseñanza básica primaria de Colombia, poniendo énfasis en la evaluación del proyecto adelantado desde el gobierno de Alfonso López con el nombre de Renovación Curricular. La evaluación incluía el programa de Escuela Nueva, en los aspectos administrativos, en la capacitación del personal docente y en el mismo componente evaluativo de la renovación curricular (UNESCO, 1986).

Aunque todo el informe evalúa y recomienda, el capítulo V está dedicado a evaluar el componente evaluativo de la Renovación Curricular en sus etapas de experimentación y generalización desde 1977 hasta 1985 y a proponer una acción integral para mejorar la evaluación en el interior del Ministerio de Educación Nacional (MEN), acción que comprendería la formación de una masa crítica de profesionales capaces de realizar «evaluaciones diagnósticas, hacer seguimientos y evaluar el impacto 
de las políticas implementadas en el sector educacional; la adopción e instalación de la evaluación como una práctica corriente en la programación del MEN (...); y la valoración de la evaluación por parte de los cuadros decisionales...» (UNESCO, 1986, p.75).

El programa de Escuela Nueva, por lo demás, ha sido objeto de varias evaluaciones realizadas por el mismo Ministerio de Educación y por el Instituto Ser de investigaciones. Ahora bien, en el período de gobierno del Presidente César Augusto Gaviria Trujillo (1990-1994), caracterizado por implementar políticas neoliberales y de apertura económica, encomendó a los diez científicos más reconocidos en el país como: Rodolfo Llinás, Manuel Elkin Patarroyo, Carlos Eduardo Vasco, Ángela Restrepo, Gabriel García Márquez, y otros, la realización de un diagnóstico de la situación del país en los aspectos de educación, ciencia y tecnología, así como a presentar una carta de navegación para la nación a fin de superar los problemas encontrados, de esta iniciativa se publicó un documento conocido como: Colombia: al filo de la oportunidad. Misión de Ciencia, Educación y Desarrollo, que aunque no evalúa la educación de los aprendizajes de los estudiantes, presenta una mirada, prospectiva de lo que debe hacerse en educación como estrategia de desarrollo nacional y este informe se ha tenido para formular el marco de la nueva constitución nacional y de igual forma en el Plan Decenal de Educación Nacional.

Los resultados del informe mencionado, están también ligados a las tendencias dominantes a nivel mundial, dado que esta comisión se asesoró por expertos internacionales en el campo de la ciencia, la tecnología y la educación, pues era urgente modernizar la educación, la ciencia y la tecnología con miras a lograr el crecimiento económico. Este documento recomienda revisar las relaciones recíprocas entre calidad y la cantidad de la educación, dado que de esta forma se podría llegar a la superación de la vaguedad de estos conceptos, tal como políticos y técnicos lo venían manejando. Consecuencia de esto, el informe plantea la reorganización de los Exámenes de Estado, vale decir, del primer examen de competencias básicas para quienes terminen el ciclo de Educación Básica y de los exámenes de ingreso a la Educación Superior. Todo con el propósito de captar información de los distintos niveles y grados de la educación formal.

Cabe aclarar que la mayor parte de las sugerencias del informe dan prioridad a la calidad de la educación en todos sus aspectos: insumos, 
agentes, ambientes y productos de todos los programas educativos. Para lograr este reto, recomiendan la creación y el fortalecimiento de un Sistema Nacional de Evaluación con capacidad de hacer los estudios teóricos, las investigaciones y evaluaciones que se requirieran, la divulgación y discusión de la información obtenida, con el propósito de que la sociedad colombiana en general estuviera informada sobre los procesos de la educación y Saber si se habían logrado los resultados proyectados. Para el momento de la presentación del informe ya se había promulgado la Ley General de la Educación o Ley 115 de 1994 y la Ley 30 de 1992 con su correspondiente Decreto Reglamentario 1860 de agosto de 1994, mediante el cual se definían algunas normas evaluatorias para la Educación Básica y Superior (Cerda y León, 2005).

Teniendo en cuenta el contexto anterior donde se muestran a grandes rasgos los orígenes de la evaluación en Colombia, se presentan a continuación las transformaciones de la misma desde la legislación colombiana con sus procesos y cambios en la educación Básica primaria y secundaria para los estudiantes. Se da comienzo a esta descripción desde las primeras pruebas que se realizan a los estudiantes que ingresan a la educación superior, de igual forma previo a las leyes de educación emitidas posteriormente por el gobierno nacional.

La primera evaluación que se aplica a los estudiantes en el país, la hace el Servicio de Admisión Universitaria y de Orientación Profesional en el año de 1968, este organismo fue el encargado también de su diseño. Este examen no fue de carácter obligatorio, tampoco diseñado de forma unificada para todas las universidades, ni mucho menos su aplicación periódica. Se organizó teniendo como base la necesidad de las universidades de hacer selección de los estudiantes aspirantes a la Educación Superior. Para el mes de septiembre de este mismo año se aplicaron los primeros exámenes unificados para toda la población colombiana, por parte del Servicio Nacional de Pruebas (SNP), antiguo Servicio de Admisión Universitaria y Orientación Profesional, quedando como dependencia del recién creado Instituto Colombiano para la Evaluación de la Educación (ICFES). Incluyó cuatro pruebas: aptitud matemática, aptitud verbal, razonamiento abstracto y relaciones espaciales. También incluyó cinco pruebas de conocimientos: ciencias sociales, filosofía, química, física, biología e inglés. Para su calificación se usaron escalas nacionales. 
En 1980 se reglamenta el Examen de Estado con el Decreto 2343 de 1980, que lo establece como oficial y obligatorio, siendo esta una prueba de carácter académico que tenía como objetivo comprobar el nivel mínimo de aptitudes y de conocimientos; fue de cobertura nacional, exigido también como requisito para el ingreso a la universidad. La prueba debía evaluar conocimientos sobre las áreas básicas comunes a las diversas modalidades de la secundaria y mediante pruebas especiales se evaluarían las habilidades, destrezas y aptitudes. Posterior a esto el Decreto 1219 de 1985, reglamenta el artículo sexto del Decreto 2343/80, donde se determina que el Examen de Estado tendría vigencia de forma indefinida.

Con la Ley 30 de 1992, con la cual se reforma la Educación Superior hoy vigente, se ratifica el Examen de Estado como requisito de obligatorio cumplimiento y pasa a ser condición para el ingreso a los diferentes programas de Educación Superior, además de poseer el título de bachiller.

Para las décadas de los ochenta y noventa el examen estaba diseñado por nuevas pruebas agrupadas así: Ciencias Naturales (Biología, Química, Física), Lenguaje ( Aptitud Verbal, Español y Literatura), Matemáticas (Aptitud Matemática y Conocimientos en Matemáticas), Ciencias Sociales (Historia, Geografía y Filosofía) y una prueba Electiva (que se podía escoger entre: Razonamiento Abstracto, Relaciones Espaciales, Razonamiento Mecánico, Inglés, Conocimientos Pedagógicos, Conocimientos Agropecuarios, Contabilidad, Comercio, Francés, Metalmecánica, Electricidad, Salud y Nutrición, Artes, Construcciones Civiles, promoción de la Comunidad, Medio Ambiente, Democracia o Etnoeducación); esta prueba tuvo modificaciones en este lapso. El resultado de esta prueba se presentaba en tres tipos así: un puntaje por prueba, otro por área y un puntaje total.

Para el año 2000 cambia el enfoque y se inicia la evaluación basada en competencias. Estos procesos de cambio se originan dadas las condiciones que ya se han mencionado como: las recomendaciones del informe de la « la misión de sabios», los cambios de las disciplinas en el contexto internacional y los nuevos modelos psicométricos para la medición y evaluación educativa, la globalización cultural, social, política y económica del nuevo contexto mundial, los avances de las pruebas de orden internacional y las investigaciones del ICFES sobre los procesos de evaluación de la calidad de la educación. Como resultado de estos 
procesos se desarrollan las pruebas que se conocen hoy como SABER, que fueron creadas en el año 2001, estableciéndose como obligatorias, dado que la política educativa de calidad en su componente de evaluación centra su atención en valorar el desarrollo de competencias básicas de los estudiantes.

Para aclarar este punto, a continuación se hace una descripción de las características de cada una de las pruebas:

\section{Pruebas Saber}

Esta prueba se convierte en una herramienta para obtener información de base censal válida y confiable para ser usada en las instituciones oficiales y privadas. También sirve para guiar la labor docente al interior de sus aulas, la revisión del currículo y hacer efectivo su proceso pedagógico.

Durante la década de los noventa se aplica la prueba por primera vez, con el propósito de evaluar las áreas críticas para el aprendizaje: Matemáticas, Lenguaje, Ciencias Naturales, Ciencias Sociales y Ciudadanía; además, de permitir un seguimiento a la evolución de los resultados y definir su permanencia y características del diseño, también permiten la divulgación de los resultados para las instituciones en cuanto a las debilidades y fortalezas para la toma de decisiones con propósitos de mejoramiento.

Esta prueba debe ser presentada por los estudiantes que finalizan quinto primaria y noveno de bachillerato porque en estos grados se culminan los ciclos de Educación Básica Primaria y Secundaria respectivamente. Tienen un carácter nacional y una periodicidad de tres años.

El Ministerio de Educación Nacional, el Instituto Colombiano para el Fomento de la Educación Superior (ICFES), hoy Sistema Nacional de Evaluación de la Educación (organismo creado en 1994) y el Servicio Nacional de Pruebas, son quienes se encargan del diseño y aplicación de estas pruebas.

Las pruebas SABER han sido aplicadas así: para los años 1992-1993 se hizo una evaluación de carácter muestral en las áreas de Matemáticas y Lenguaje para los grados 3ro., 5to., 7mo. y 9no. En el periodo 1997-1998 también se aplica de forma muestral en las mismas áreas a los grados 
en los niveles antes mencionados. En los años 2002-2003 se aplica una prueba censal y muestral cuasi-controlada en los departamentos de Antioquia, Nariño y Bogotá, donde las áreas evaluadas fueron: Matemáticas, Lenguaje, Ciencias Naturales y Competencias Ciudadanas, para los grados quinto y noveno. En el 2005-2006, se hace una aplicación censal del 90\% y 10\% muestral controlada, en áreas como Matemáticas, Lenguaje, Ciencias Sociales y Competencias Ciudadanas, a los grados $5^{\circ}$ y $9^{\circ}$ (Fernández, 2005, p. 6).

Si bien el país cuenta con una cantidad considerable de información sobre el desempeño de los estudiantes, sucesivas variaciones en el enfoque y metodología de las pruebas impiden hacer comparaciones rigurosas sobre cómo ha sido la evolución en el tiempo. No obstante, es posible inferir algunos comportamientos regulares en términos de las competencias desarrolladas por los estudiantes en lenguaje y matemáticas en los distintos ciclos educativos. Las evaluaciones en el área de lenguaje se han concentrado especialmente en establecer la capacidad de los estudiantes para comprender distintos tipos de textos y relacionarlos entre sí para construir nuevos conocimientos. En matemáticas, el énfasis ha estado en la capacidad para resolver problemas que involucran conceptos y lenguajes propios del área en distintos niveles de complejidad. En Lenguaje, tanto en quinto como en noveno grado, el porcentaje de estudiantes que no alcanza el nivel mínimo de logro ha disminuido consistentemente. La mayoría de estudiantes de quinto logra y supera los niveles mínimos de lectura literal (nivel B) y un porcentaje importante también supera el nivel medio de lectura inferencial (nivel C). No obstante, solamente la mitad (52\%) de los estudiantes logra y supera el nivel de mayor complejidad (nivel D), necesario para dar significado e interpretar los textos más allá del simple seguimiento lineal del mismo y para lograr la interacción entre el lector y el texto, poniendo en práctica los Saberes sobre lenguaje, literatura y otras disciplinas. A su vez, en noveno el porcentaje de estudiantes que logran y superan el nivel de lectura literal (nivel C) es mayor que en quinto, como es de esperarse, y una proporción importante se centra en los niveles medios; aunque un menor número (4\%) alcanza el nivel de mayor complejidad. Los resultados de la prueba de lenguaje al terminar la educación media confirman esta tendencia: la mayoría de los estudiantes presenta un nivel de desempeño intermedio, y solamente un porcentaje menor al 10\% está por encima de los niveles altos complejidad (Plan Sectorial-Revolución Educativa, 2006-2010, p.24). 
En matemáticas la situación es similar: el porcentaje de estudiantes que no alcanzan el nivel mínimo también ha disminuido en los últimos años, pero es más alto que en lenguaje. Tanto en quinto como en noveno grado hay una concentración en los niveles de menor complejidad, donde los problemas planteados se refieren a situaciones cotidianas. En quinto grado el nivel B - resolución de problemas rutinarios que requieren tan sólo de una operación o una relación para su resolución - es alcanzado y superado por cerca del $90 \%$ de los estudiantes. A su vez, en noveno grado, donde se requiere mayor competencia y la resolución de problemas más complejos, un porcentaje inferior al de quinto grado alcanza el nivel mínimo de desempeño $(C)$ y en los niveles intermedios y altos se observa una participación muy reducida Esta situación se replica en la prueba de matemáticas del examen de Estado, en la que la mayoría de los estudiantes se ubica en el rango medio de desempeño (93\% en calendario A y $96 \%$ en B). Esto quiere decir que logran abordar algunos aspectos básicos de la matemática escolar y enfrentan exitosamente situaciones que contienen elementos no rutinarios que les exigen relacionar diferente información, pero sin alcanzar los niveles de mayor complejidad. El 80\% de los estudiantes de calendario A, se ubica en el nivel intermedio de la competencia de comunicación y representación; el 68\% está en el nivel intermedio de la competencia de razonamiento y argumentación, y el 90\% está en los niveles bajo y medio de la competencia de modelación, planteamiento y resolución de problemas. Los resultados para calendario B siguen una tendencia similar (Plan Sectorial-Revolución Educativa, 2006-2010, p. 28).

\section{Pruebas Saber II}

Para la Educación media o secundaria, las Pruebas SABER 11 (anteriormente llamado Examen de Estado), deben ser presentadas por los estudiantes que terminan el 11avo grado y aquellos estudiantes que deseen ingresar a la educación superior. Esta prueba evalúa las competencias básicas de Matemáticas, Lenguaje, Geografía, Filosofía, Historia, Biología, Química, Física e Idioma Extranjero. También evalúa el Componente Flexible, de escogencia libre por el estudiante, el cual corresponde a los temas de Violencia y Sociedad, Medios de Comunicación, Cultura y Medio Ambiente (Vélez, 2010).

El Examen de Estado de la Educación Media, (ICFES o SABER 11º), es un instrumento estandarizado para la evaluación externa, que conjunta- 
mente con los exámenes que se aplican en los grados $5^{\circ}, 9^{\circ}$ y al finalizar el pregrado, hace parte de los instrumentos que conforman el Sistema Nacional de Evaluación.

La estructura esencial del Examen se mantendrá por lo menos doce (12) años a partir de su adopción por la Junta Directiva y de su aplicación a la población, sin perjuicio de que puedan introducirse modificaciones que no afecten la comparabilidad de los resultados en el tiempo. El ICFES dirigirá y coordinará el diseño, la producción y la aplicación de las pruebas y el procesamiento y análisis de los resultados del Examen, para lo cual podrá apoyarse en las comunidades académicas y profesionales. El calendario de aplicación será determinado por el ICFES, de acuerdo con el reporte sobre la población que cumpla el requisito para presentar el Examen establecido en el decreto que lo reglamenta.

Estas pruebas, tienen por objetivo, según lo dispuesto por el Decreto 869 de 2010, entre otros los siguientes:

Comprobar el grado de desarrollo de las competencias de los estudiantes que están por finalizar el grado undécimo de la educación media; proporcionar a las instituciones educativas información pertinente sobre las competencias de los aspirantes a ingresar a programas de educación superior, así como sobre las de quienes son admitidos, que sirva como base para el diseño de programas de nivelación académica y prevención de la deserción en este nivel; monitorear la calidad de la educación de los establecimientos educativos del país, con fundamento en los estándares básicos de competencias y los referentes de calidad emitidos por el Ministerio de Educación Nacional; servir como fuente de información para la construcción de indicadores de calidad de la educación, así como para el ejercicio de la inspección y vigilancia del servicio público educativo y ofrecer información que sirva como referente estratégico para el establecimiento de políticas educativas nacionales, territoriales e institucionales (Decreto 869 de 2010).

Los resultados del examen de Estado por niveles de competencia de 2006 también son coherentes con los resultados de las Pruebas Saber, ya mencionados. En la acción de construir el sentido del texto, el 31\% de los estudiantes se queda en la comprensión de la información explícita, el 61\% realiza lecturas relacionales e inferenciales, y sólo un 8\% puede evaluar el texto de manera crítica. En la tarea de contextualizar 
y dar explicación a las ideas que articulan y dan sentido a un texto, el $25 \%$ lo hace desde esquemas muy cotidianos o inmediatos al texto; un $55 \%$ busca relacionar información del escrito con otros Saberes para dar sustentaciones más complejas y un 20\% logra la construcción de un punto de vista propio a partir del texto. Finalmente, en cuanto a la acción de plantear alternativas frente a las situaciones presentes en un texto, el 62\% lo hace desde información explícita, el 27\% busca relacionar información para hallar respuestas más complejas y sólo el 11\% lo hace desde una evaluación crítica del contenido. Los datos disponibles sobre comprensión de lectura en educación superior sugieren una evolución hacia la comprensión en niveles de interpretación textual o crítica que supera lo alcanzado en la educación básica y media (MEN, 2008).

Teniendo en cuenta lo anteriormente descrito, se puede ver el sentido real de las pruebas aplicadas hasta la fecha pero también, dándosele importancia a la evaluación como uno de los factores de calidad, obteniendo información a partir de los resultados, de los desempeños académicos de los estudiantes, dichos elementos se constituirían posteriormente en información importante para que el Ministerio de Educación Nacional pueda seguir trabajando en el mejoramiento de la calidad de la educación básica colombiana y cómo trabajar mas coherencia para el ingreso a la educación superior.

Veamos, entonces cómo han venido siendo las aplicaciones de las pruebas en la educación superior:

\section{Exámenes de Estado de Calidad de Educación Superior-ECAES o Saber Pro}

Para el caso de la Educación Superior, el ICFES, también ha diseñado una prueba para articular la Educación Básica y Media con la Educación Superior que denominó inicialmente como Examen de Calidad de la Educación Superior (ECAES), hoy llamado SABER PRO, cuyo propósito es evaluar las competencias y conocimientos de las diferentes profesiones. La primera aplicación de esta prueba, con esta nueva denominación, se hizo en septiembre de 2010.

Desde sus inicios ha contado con la participación activa de la comunidad académica y se formalizó en el 2003 cuando el Ministerio de Educación y el ICFES, realizaron una convocatoria nacional en la que invitó a to- 
das las instituciones de educación oficiales y privadas, asociaciones de facultades y agrupaciones de profesionales para presentar proyectos de diseño y elaboración de estos exámenes. En junio del 2003, el gobierno expidió el Decreto 1781 que reglamentó los Exámenes de Calidad de la Educación Superior, conocidos como ECAES.

Los ECAES o pruebas Saber Pro, cumplen varios objetivos, entre los cuales se encuentran: Comprobar el grado de desarrollo de las competencias de los estudiantes de último grado de pregrado, Servir como fuente de información para la construcción de indicadores de calidad educativa, Proporcionar información para alimentar los procesos de mejoramiento de la calidad en las instituciones de educación superior, Proveer criterios para la autoevaluación institucional, pues permiten identificar fortalezas y debilidades de los programas y constituyen un insumo importante para la revisión de planes del currículo².

El ECAES, evalúa competencias interpretativas, argumentativas y propositivas. Estas competencias corresponden a 8 áreas del conocimiento que toma como base las características y particularidades de cada programa académico. Las pruebas de cada programa tienen dos componentes transversales comunes: comprensión lectora e inglés. Actualmente, se aplican una vez al año y son obligatorias para todos los estudiantes.

Los programas de Ciencias son los que presentan los mejores promedios, seguidos por Ciencias de la Salud. Las Ciencias de la Educación y el área de Economía y Administración conforman el tercer grupo y los programas técnicos y tecnológicos el cuarto grupo, con menores promedios. No obstante, las carreras técnicas y tecnológicas presentan una mejoría con respecto a años anteriores, mientras que las áreas como ciencias naturales y de la salud han desmejorado sus resultados en esta materia (MEN, 2008).

Con lo anterior, Colombia demuestra que viene trabajando en la conformación de un sistema de evaluación para los estudiantes de Educación Básica, Media y Superior organizado desde el Ministerio de Educación con el apoyo de ICFES, organismo encargado del diseño, aplicación y divulgación de dichas evaluaciones.

2 Se recomienda ampliar esta información en el documento: Guzmán, Serna \& Hoyos (2012). 


\section{Pruebas Internacionales}

En referencia a las pruebas internacionales, Colombia ha participado en evaluaciones internacionales con el propósito de tener un nivel comparativo de su rendimiento con la de otros países y mostrar un compromiso con la evaluación y su desempeño. Estas también le han permiten valorar la importancia de la evaluación en el debate educativo mundial, de igual forma le permiten ver las metodologías y las comparaciones entre países y en períodos de tiempo (muestras, instrumentos, técnicas de procesamiento y análisis de información) y estudios innovadores en cuanto a enfoques, campos y técnicas de evaluación.

Dichas pruebas tienen que ver con las áreas de Lenguaje y Matemáticas principalmente. Para el año 1997, el país participó en el primer Estudio Internacional Comparativo de Lenguaje, Matemáticas y Factores Asociados con los grados tercero y cuarto de primaria aplicado por el Laboratorio Latinoamericano de Evaluación para la Calidad de la Educación (LLECE), dirigido por la UNESCO para América Latina, mostrando un desempeño muy parecido con los países que salieron bien favorecidos, como Argentina, Brasil, Chile y Cuba. Para el año 1995- 2000, el país también hace parte del Estudio Internacional de Educación Física, desarrollado en 1999. De igual manera participa el año 2002 en el Primer Estudio Internacional del Progreso de la Lectura (PIRLS), aplicado por la Agencia Internacional de Evaluación de Logro Educativo (IEA). Su participación reciente en pruebas internacionales fue, en los años 2006 y 2008, en el Programa de Evaluación Internacional de Estudiantes (PISA) y en el Estudio Internacional de Tendencias en Matemáticas y Ciencias (TIMSS) en el año 2007; también participó en los años 2005 y 2006 en el Estudio Regional Comparativo y Explicativo (SERCE).

En estas pruebas los resultados han mostrado un desempeño bajo y dejan en evidencia la enorme distancia que separa a Colombia con respecto a los países desarrollados e incluso con algunas naciones de la región (Banco Mundial Colombia, 2008).

El trabajo que se viene haciendo desde el Ministerio de Educación Nacional a partir de la organización de las pruebas anteriormente mencionadas, no se ha quedado solo en a evaluación de los estudiantes, cuando culminan los niveles establecidos para la educación básica primaria, secundaria y superior, también se vienen desarrollando estrategias 
que le permitan coherencia con el sistema de evaluación al interior de las aulas como se podrá mostrar en la breve descripción de la misma a continuación.

\section{La evaluación de los aprendizajes al interior de las aulas}

De acuerdo a lo expuesto en el Portal Colombia Aprende del Ministerio de Educación Nacional, desde los años sesenta y parte de los setenta las políticas de evaluación y promoción de estudiantes al interior de las instituciones de educación básica y media o secundaria, establecían la necesidad de centrar los esfuerzos en la definición de promedios sumativos de calificaciones en escala numérica de manera periódica. En esa época el país contaba con un currículo preestablecido de orden nacional y se emprendían procesos evaluativos de orden netamente cuantitativo.

Según el informe de la Organización de Estados Iberoamericanos (OEI), a partir de la expedición de la Ley General de Educación (1994) se da una profunda reforma a la educación. Se establece el Proyecto Educativo Institucional (PEI), como la carta de navegación de las instituciones educativas, permitiendo así contemplar las diferencias socioculturales del país. Esto implicaba el cambio de un currículo centralizado, único, basado en objetivos, hacia la definición de currículos más pertinentes, en el marco de una política general que buscaba desarrollar competencias. En este sentido, la evaluación ya no sería sumativa (promedio de calificaciones numéricas por períodos lectivos), sino formativa, integral y cualitativa. Ello condujo a la expedición de las normas sobre el tema, tales como el Decreto 1860 de 1994, y los Decretos 230 y 3055 de 2002. En dichas normas en particular en el Decreto 1860 de 1994, que luego es derogado por el 230 de 2002, se comienzan a constituir comisiones de evaluación con el fin de apoyar las acciones tendientes a la recuperación de los logros de estudiantes y a su vez definir las actividades curriculares. Para cumplir con este objetivo, era necesario entregar informes descriptivos de los logros y dificultades en lugar de las calificaciones numéricas, como se venía haciendo.

Por su parte, el Decreto 230 de 2002, recogió el espíritu del proceso de evaluación consagrado en el Decreto 1860 de 1994, con adiciones tales como la determinación de cuatro (4) periodos académicos durante el año escolar. Al mismo tiempo serviría como referente para el informe final integral de evaluación de cada año lectivo, que ya no solamente 
sería descriptivo sobre logros y dificultades sino también expresados en una escala cualitativa de valoración: excelente, sobresaliente, aceptable, insuficiente y deficiente.

De otra parte, el Decreto 3055 de 2002, modificó el artículo $9^{\circ}$ del Decreto 230 de 2002, en el sentido de que los establecimientos educativos debían garantizar un mínimo de promoción del 95\% del total de los educandos que finalizaran el año escolar en la institución educativa. Este criterio de promoción del $95 \%$ ha tenido fuertes implicaciones para las decisiones de promoción de grado, toda vez que, determinadas instituciones educativas se ven obligadas a promover de un grado a otro a estudiantes con evaluación insuficiente en tres o más áreas, debiendo cumplir con actividades de evaluación de recuperación a más tardar una semana antes de iniciar el siguiente año lectivo, como lo señala el artículo 10 del Decreto 230 de 2002 (MEN, 2008).

Es así como se empieza a concebir la educación desde el marco de las competencias y por ende la evaluación al interior de las prácticas de aula cobra un sentido distinto. Se habla entonces de una evaluación que se abre hacia la valoración, no solamente de los avances del estudiante en su formación integral, sino que también involucra a los distintos actores educativos de una forma comprometida y dinámica. Con esto los padres de familia, los docentes, los directivos docentes, la institución y el sistema educativo mismo, adquieren un protagonismo esencial en los procesos evaluativos de los estudiantes y en el acompañamiento de estos para el logro de las competencias básicas.

Para el año 2008 se abre la discusión sobre la evaluación al interior de las aulas y se plantea la necesidad de revisar el sistema de evaluación vigente y su promoción automática. Hecho que ha causado polémica en el sector educativo colombiano, concluyéndose que el mayor problema que tiene la educación es el sistema de evaluación, lo que ha llevado a que los ciudadanos colombianos popularmente expresen que «el proceso de enseñanza- aprendizaje se encuentra en un estado de relajación». Por tal motivo se impulsa durante este año una amplia reflexión sobre el Decreto 230 de 2002, dado que se quería derogar el tema de la promoción automática y modificar el sistema de evaluación.

Consecuencia de esto, el Decreto 1290 de 2009, recogió esta discusión y planteó la autonomía de las instituciones en la formulación de los 
sistemas de evaluación y su escala de valoración equivalente con la escala de valoración nacional, con el propósito de permitir la movilidad de estudiantes en ámbito nacional; esta escala está determinada con los siguientes desempeños: desempeño superior, desempeño alto, desempeño básico y desempeño bajo.

Para la implementación de este Decreto se estableció el desarrollo de talleres informativos en las 92 secretarías municipales, 7.500 establecimientos educativos de educación al igual que 25 talleres regionales con acompañamiento virtual. El Decreto entró en vigencia al inicio del año escolar 2009 para las instituciones de calendario B y el primero de enero de 2010 para los establecimientos de calendario A. En el Foro Educativo Nacional de este mismo año, denominado «Evaluar es Valorar» se hace su lanzamiento. Esta decisión se toma dada la importancia que tiene el tema de la Evaluación en el Sistema Educativo. De acuerdo a los lineamientos del Ministerio de Educación Nacional, la Ministra de Educación, expresó que:

La evaluación no debe ser objeto de control, jerarquización y exclusión de quienes participan en el sistema y por el contrario debe cumplir su papel pedagógico social amplio e incluyente, al igual que debe promover los aprendizajes de forma transcendental para formar personas integrales, ciudadanos competentes y comprometidos con el bien común (Vélez, 2010, p. 2).

La gran consulta que hace el Ministerio de Educación en el año 2007, para la formulación del segundo Plan Decenal de Educación 2006-2015, se muestra la Evaluación como el factor de mayor problemática en la comunidad educativa, en especial en lo que respecta a la promoción de los estudiantes de la Educación Preescolar, Básica y Media; por tanto para el 2008. Promovió una movilización en todo el país, para escuchar la opinión de todos los actores sobre el tema. La enseñanza de esta movilización, dio como resultado aportes, recomendaciones, inquietudes y sugerencias que se convierten en el insumo de las áreas temáticas de este foro para seguir alimentando esta discusión. Es por esto que el foro se constituyó en el espacio que permitió el cuestionamiento de los aciertos, la ampliación de los consensos y la continuación de esta reflexión, la cual permitirá el avance y formulación de una nueva política pública en el tema de la evaluación, útil para los estudiantes Colombianos de la Básica Primaria y Secundaria. 


\section{Conclusiones}

En el mundo, la evaluación tuvo sus orígenes en Estados Unidos e Inglaterra, sin embargo, se han desarrollado avances y transformaciones en los procesos, los mecanismos y los instrumentos de evaluación en diferentes países, con el fin de contar con herramientas que midan de forma efectiva la calidad del aprendizaje y, por ende, de la educación. Este tema, por tanto, ocupa un lugar estratégico y preponderante en las políticas de calidad, descentralización y profesionalización de los sistemas educativos en la mayoría de los países del mundo. Algunos aspectos adicionales a la evaluación del aprendizaje están centrados en su uso sistemático y en la ampliación del campo de aplicación de la evaluación hacia territorios que antes no eran evaluados: la concepción y desarrollo del currículo, los centros educativos, el profesorado y la propia administración de la educación.

Gracias a las recomendaciones hechas en el documento elaborado en el Informe Misión de Sabios. Colombia: Al Filo de la Oportunidad. Misión Ciencia, Educación y Desarrollo, se definieron los lineamientos para hacer evaluaciones que midieran los aprendizajes obtenidos en la educación básica y secundaria. Esto se constituyó en los orígenes en Colombia de toda la dinámica tendiente al diseño de las pruebas que hoy se conocen como Saber, Saber 11 y Saber Pro.

En la actualidad, Colombia ya cuenta con una línea de base censal compuesta por información válida que está siendo utilizada por las instituciones educativas colombianas, como referente para su mejoramiento continuo, lo que conlleva a la definición o redefinición de sus planes de mejoramiento. Esto permite que todos los miembros de la comunidad educativa (estudiantes, docentes, padres de familia, directivos, entre otros) puedan hacer sus aportes dentro del proceso educativo, cumpliendo con el rol que les corresponde $y$, de esta forma, se logre una participación integral. Además, cabe anotar que la política educativa de calidad ha centrado su atención en evaluar el desarrollo de las competencias básicas de los estudiantes en áreas tales como matemáticas, lenguaje, ciencias naturales y competencias ciudadanas, lo que ha permitido establecer en qué medida se ha dado el desarrollo de estas competencias en diferentes niveles de formación. 


\section{Referencias}

Aldana, E., Chaparro, L., García, G., Gutiérrez, R., Llinás, R., Palacios, M., Patarroyo, M, Posada, E, Restrepo, A. \& Vasco, C. (1996). Colombia: Al Filo de la Oportunidad. Misión Ciencia, Educación y Desarrollo. Tomo I. Bogotá: Tercer Mundo Editores.

Banco Mundial Colombia (2008). La calidad de la Educación en Colombia: Un análisis y algunas opciones para un Programa de Política. Unidad de Gestión del Sector de Desarrollo Humano. Oficina Regional de América Latina y del Caribe.

Brunner. J. (2002). Educación: Escenarios de Futuro. Nuevas Tecnologías y Sociedad de la Información. Santiago de Chile: Preal.

Campbell, D. T. (1969). Reform as experiments. En: American Psychologist, no. 4, 24.

Cerda, H. y León, A. (2005). La evaluación en la educación Colombiana. A propósito de un estudio sobre la realidad evaluativa en la Universidad Cooperativa de Colombia. Bogotá: Comité de investigaciones CIFE. Facultad de Educación-Escuela de Posgrados.

Constitución Política de Colombia de 1991. Presidencia de la República.

Correa, S., Puerta, A. \& Restrepo, B. (2002) Investigación Evaluativa. Programa de Especialización En: Teoría, Métodos y Técnicas de Investigación Social. Bogotá. Instituto Colombiano para el Fomento de la Educación Superior. pp. 37-39.

Darling- Hammond, L. (2004). Standards, Accountability and School Reform. En: Teachers College Record.

Decreto 1219 de abril 30 de 1985. Por el cual se adopta una determinación sobre los Exámenes de Estado para el ingreso a la educación superior. Diario oficial N³6977 de 17 de mayo de 1985. Bogotá.

Decreto 1860 de agosto 3 de 1994. Por el cual se reglamenta parcialmente la Ley 115 de 1994, en los aspectos pedagógicos y organizativos generales. Ministerio de Educación Nacional. Bogotá.

Decreto 230 de febrero 11 de 2002. Por el cual se dictan normas en materia de currículo, evaluación y promoción de los educandos y evaluación institucional. Recuperado de www.alcaldiabogota.gov. co/sisjur/normas/Norma1.jsp? $\mathrm{i}=4684$ 
Decreto 2343 de 30 de 1985. Mediante el cual se reglamenta los Exámenes de Estado para Ingreso a la Educación Superior. Presidencia de la República. Diario oficial N³6977 de mayo de 1985.

Decreto 3055 diciembre 12 de 2002. Por el cual se adiciona el artículo $9^{\circ}$ del Decreto 230 de 2002. Ministerio de Educación Nacional. Bogotá.

Decreto 869 de Marzo 17 de 2010. Por el cual se reglamenta el Examen de Estado de la Educación Media, ICFES SABER 11². Presidencia de la República. Bogotá.

Decreto No. 1290 de 16 Abril de 2009. Por el cual se reglamenta la evaluación del aprendizaje y promoción de los estudiantes de los niveles de educación básica y media. Ministerio de Educación Nacional. República de Colombia, Bogotá.

Decreto Número 1781 de junio 26 2003. Por el cual se reglamentan los Exámenes de Estado de Calidad de la Educación Superior, ECAES, de los estudiantes de los programas académicos de pregrado. Congreso de la República. Bogotá.

Drucker, P. F. (1996). La sociedad del poscapitalista. Bogotá: Grupo Editorial Norma.

Fernández, G.H. (2005). ¿Cómo interpretar la evaluación pruebas Saber? Subdirección de Estándares y Evaluación. Bogotá: Ministerio de educación Nacional.

Foro Educativo Nacional (2008). Discusión Nacional: La Evaluación en Colombia - Documento Síntesis - Foros Regionales, Movilización Plan Decenal, Talleres de Discusión Nacional. Bogotá. 57 p.

Grossman, J. B. (1994). Evaluating Social Policies: Principles and U.S. Experience. En: Oxford Journals. The World Bank Research Observer. [en línea] http://wbro.oxfordjournals.org/content/9/2/159.short

Guzmán. C. T., Serna, C. \& Hoyos, D. F. (2012). Las pruebas ECAES en Colombia: una evaluación a la evaluación. Bogotá: Universidad Central de Bogotá.

Instituto Colombiano de Pedagogía (ICOLPE). (1972). Lecturas sobre definición del concepto de evaluación; metodología de evaluación institucional; definición de evaluación educacional. Bogotá: Centro de Documentación e Información Pedagógica, CENDIP, 1973, 1974, 1975. 
Ley 115 de febrero 8 de 1994. Por la cual se expide la ley general de educación. Diario Oficial No. 41.214, de 8 de febrero de 1994. Presidencia de la República. Bogotá.

Ley 30 de Diciembre 28 de 1992. Por la cual se organiza el Servicio Público de la educación Superior. Diario Oficial 40.700. Bogotá.

López R. F. (1997). La gestión de calidad en educación. Madrid: La Muralla.

Madauss, G. F. \& Linnan, R .(1983). The Outcome of Catholic Education? School Review.

Ministerio de Educación Nacional. (2008.) Plan Sectorial Revolución Educativa.2006-2010. Revolución Educativa Colombia Aprende. Documento $N^{\circ}$ 8. Bogotá. Recuperado de www.mineducación.gov.co.

Ministerio de Educación Nacional. (s.f.). Sistemas de Información. Recuperado el 4 de Enero de 2011 del sitio web del Ministerio de Educación Nacional: http://www.mineducacion.gov.co/1621/propertyvalue-37666.html

Newman, J., Rawlings, L., \& Gertler, P. (1994). Using randomised control designs in evaluating social sector programs in developing countries. En: The World Bank Research Observer.

Peña, M. (2008). Las evaluaciones externas en Colombia. Evolución y Balance. Bogotá: ICFES.

Plan Decenal de Educación 2006-2016. Pacto Social por la Educación, Organización de Estados Iberoamericanos para la Educación la Ciencia y la Cultura. Evaluación de aprendizajes en Colombia. Recuperado de www.plandecenal.edu.co.

Portal Colombia Aprende. Ministerio de Educación Nacional. Consultado en Noviembre 1 de 2010 en: http://www.colombiaaprende.edu.co/ html/home/1592/article-219406.html

Posada, W.Y., Martínez, J. A. y Ruiz, L.A. (2010). La evaluación de la Educación Física y las políticas educativas en Colombia. Avatares entre la mente, el papel y el contexto. El caso de Manizales y Villamaría (Caldas). En: Revista Iberoamericana de Educación. n. 53/1. Organización de Estados Iberoamericanos para la Educación, la Ciencia y la Cultura (OEI). Departamento de Estudios Educativos. Universidad de Caldas, Colombia. 
Ravela, P. (2001). Los próximos pasos: żcómo avanzar en la evaluación de aprendizajes en América Latina? Santiago de Chile: PREAL.

Restrepo, B. (1974). Conferencias sobre evaluación. Documento mimeografiado. Medellín: Facultad de Educación, Universidad de Antioquia.

Stufflebeam, D. (1997). The CIPP Model for Evaluation. Draft of Chapter 6. of a new book on evaluation. Kalamazoo: The Evaluation Center. Western Michigan University.

Stufflebeam, D. \& Shinkfield, A. (1995). Evaluación sistemática - Guía teórica y práctica. España: Centro de Publicaciones del Ministerio de Educación y Ciencia, Ediciones Paidós Ibérica.

Suchman, E. (1967). Evaluative Research. New York: Russell Sage Foundation.

UNESCO. (1986). Colombia: aspectos de la calidad de la enseñanza básica primaria. París: UNESCO.

Vélez, C. M. (2010). Balance de Gobierno 2002-2010. Balance de la Revolución Educativa 2002-2010. Recuperado de http://www.mineducacion.gov.co/1621/articles-231469_archivo_pdf_discurso_arreglado.pdf.

Worthen, J., Sanders, J., \& Fitzpatrick, J. (1997). Program Evaluation. New York: Longman Publishers. 